\title{
Phase behaviour of charged colloidal sphere dispersions with added polymer chains
}

\author{
Andrea Fortini ${ }^{1}$, Marjolein Dijkstra ${ }^{1}$ and Remco Tuinier ${ }^{2}$ \\ ${ }^{1}$ Soft Condensed Matter, Debye Institute, Utrecht University, Princetonplein 5, 3584 \\ CC Utrecht, The Netherlands \\ ${ }^{2}$ Forschungszentrum Jülich, Institut für Festkörperforschung, 52425 Jülich, Germany.
}

\begin{abstract}
We study the stability of mixtures of highly screened repulsive charged spheres and non-adsorbing ideal polymer chains in a common solvent using free volume theory. The effective interaction between charged colloids in an aqueous salt solution is described by a screened-Coulomb pair potential, which supplements the pure hardsphere interaction. The ideal polymer chains are treated as spheres that are excluded from the colloids by a hard-core interaction, whereas the interaction between two ideal chains is set to zero. In addition, we investigate the phase behaviour of charged colloidpolymer mixtures in computer simulations, using the two-body (Asakura-Oosawa pair potential) approximation to the effective one-component Hamiltonian of the charged colloids. Both our results obtained from simulations and from free volume theory show similar trends. We find that the screened-Coulomb repulsion counteracts the effect of the effective polymer-mediated attraction. For mixtures of small polymers and relatively large charged colloidal spheres, the fluid-crystal transition shifts to significantly larger polymer concentrations with increasing range of the screenedCoulomb repulsion. For relatively large polymers, the effect of the screened-Coulomb repulsion is weaker. The resulting fluid-fluid binodal is only slightly shifted towards larger polymer concentrations upon increasing the range of the screened-Coulomb repulsion. In conclusion, our results show that the miscibility of dispersions containing charged colloids and neutral non-adsorbing polymers increases, upon increasing the range of the screened-Coulomb repulsion, or upon lowering the salt concentration, especially when the polymers are small compared to the colloids.
\end{abstract}

\section{Introduction}

Adding non-adsorbing polymers to colloidal dispersions allows modifying the range and strength of attraction of the effective interactions between the colloidal particles. Adjusting the range of the attraction enables manipulating the topology of the phase diagram of a colloid-polymer mixture 1, 2, 3, 4. Both the nature of the demixed phases as well as the colloid and polymer concentrations at which demixing takes place depend on the range of attraction [5]. Industrially, it is relevant to understand the phase behaviour of colloid-polymer mixtures because colloidal particles and polymer chains are often jointly present in various products, such as food dispersions [6, 7]. Theoretically, the focus has been mainly on hard spheres dispersed in polymer solutions. This is due 
to the fact that the hard-sphere dispersion is relatively simple and well-understood. Moreover, hard sphere-like colloids can actually be synthesised chemically. Examples are, for instance, stearyl-silica colloids [8] or PMMA-PHSA particles [9].

In practice, many stable dispersions containing spherical colloids consist of particles that are not 'pseudo-hard' but which can be characterised by a pair potential containing an additional soft repulsive tail. An example is a stable dispersion of charged colloids in an aqueous salt solution for which the interactions are described by the DerjaguinLandau-Verwey-Overbeek (DLVO) theory [10]. This theory predicts that the effective pair interaction between charged colloids consists of a hard-core repulsion due to the finite size of the colloids and a screened-Coulomb (Yukawa) repulsion with the screening length given by the Debye length $\kappa^{-1}$ of the solvent. The screening length $\kappa^{-1}$ defines the thickness of the double layer of opposite charge surrounding each colloid. The range $\kappa^{-1}$ of the screened-Coulomb repulsion is a function of the salt concentration of the solvent, the dielectric constant and the temperature. Adjusting the salt concentration may influence the stability of a dispersion of charged colloids mixed with a neutral depletion agent in a common aqueous salt solution [6, 11, 12, 13. Hebert [1] studied the precipitation of the charged, rod-like tobacco mosaic virus (TMV) by adding the neutral polymer polyethylene glycol (PEG). At similar PEG concentrations, precipitation of TMV was enhanced by adding salt. Patel and Russel [14] studied the phase behaviour of mixtures of charged polystyrene latex colloids and dextran as (neutral) polymer chains and reported a significant shift towards higher polymer concentrations of the fluidfluid binodal curve as compared to predictions for neutral polymer chains mixed with hard spheres. Grinberg and Tolstoguzov [6] presented generalised phase diagrams of proteins mixed with neutral non-adsorbing polysaccharides in aqueous salt solutions. The miscibility or compatibility was shown to increase when the ionic strength of the solvent was lowered. The compatibility especially increased below $0.5 M$. Finet and Tardieu [12] studied the stability of solutions of the lens protein crystalline. Adding an excess of salt to this system does not destabilise the protein dispersion. Hence, it follows that the effective attractions between the proteins are absent or are very weak in the case of screened charges. Adding PEG however induces significant attractions [12, and results in a shift of the liquid-liquid phase transition to higher temperatures [15. Adding excess salt and PEG induces instant phase separation [12. A similar synergetic effect of salt and PEG was found in aqueous solutions of (spherical) brome mosaic virus particles 13. In conclusion, the trend found in experimental studies on mixtures of charged 'colloids' plus neutral polymers is that the miscibility is increased upon decreasing the salt concentration, i.e., increasing the range of the screened-Coulomb repulsion.

In the light of these findings it is important to study theoretically mixtures of colloids with a screened-Coulomb repulsion mixed with neutral polymer chains and to investigate whether the trend found in many experimental studies is recovered. The amount of theoretical work performed so far is rather limited. Ferreira et al. [16] made a PRISM analysis up to the level of the pair interaction and computed gas-liquid spinodal curves from the effective colloid-colloid structure factor. Denton and Schmidt 
17. proposed a simple theory yielding the gas-liquid binodal curve. The fluid-solid coexistence curves were not considered and none of these theories were tested against computer simulations. Here we study the effect of a screened-Coulomb interaction on the total effective colloid-colloid interaction and on the resulting gas-liquid and fluid-solid phase transitions in a charged colloid dispersion with added non-adsorbing polymers. We demonstrate the fluid-solid coexistence is especially sensitive to the screened-Coulomb repulsion. The outline is as follows. First, in section 2 we calculate the total effective pair potential by juxtaposing the depletion and the DLVO (screened-Coulomb repulsion) contributions. In section 3, a simple Ansatz for calculating the phase behaviour of the charged colloid dispersion is addressed, followed by an analysis of the effect of nonadsorbing polymer using free volume theory in section 4.1. The theoretical results are compared with Monte Carlo simulation results in section 4.2. This communication will be summarised with some concluding remarks.

\section{The model}

We consider a suspension of charged colloidal spheres immersed together with nonadsorbing polymer in a common solvent. As the differences in length- and time-scales between the solvent molecules and the colloids and polymers are large, we can regard the solvent to be an inert continuum. Charged-stabilized colloidal suspensions can be described by a mixture of $N_{c}$ colloidal particles with charge $-Z e$, with $e$ the elementary charge, $Z N_{c}$ counterions with charge $+e$ and by pairs of salt ions of charge $\pm e$. It is convenient to consider the system in equilibrium with a salt reservoir with density $\rho_{s}^{r}$. However, computer simulations of this model are prohibited by slow equilibration, due to a large number of small counterions and salt pairs compared to the number of colloidal particles. A more coarse-grained model can be obtained by formally integrating out the degrees of freedom of the co- and counterions in the partition function [18. The effective pair interaction between the charged colloidal spheres with hard-core diameter $\sigma_{c}$, reads

$$
U_{c c}\left(R_{i j}\right)=\left\{\begin{array}{ll}
\infty & \text { for } R_{i j}<\sigma_{c} \\
\epsilon\left(\frac{\exp \left(-\kappa \sigma_{c}\left(R_{i j} / \sigma_{c}-1\right)\right)}{R_{i j} / \sigma_{c}}\right) & \text { otherwise }
\end{array},\right.
$$

where $R_{i j}=\left|\mathbf{R}_{i}-\mathbf{R}_{j}\right|$ and $\mathbf{R}_{i}$ are the positions of the centres of the colloids. The range of the repulsive tail is set by the inverse Debye screening length $\kappa \sigma_{c}=\sqrt{8 \pi l_{B} \sigma_{c}^{2} \rho_{s}^{r}}$, which is related to the salt concentration $\rho_{s}^{r}$ in the reservoir, and to the Bjerrum length $l_{B}=e^{2} / \epsilon_{s} k T$ with $k$ Boltzmann's constant, $T$ the temperature, and $\epsilon_{s}$ the dielectric constant of the solvent. The strength of the repulsion of the repulsive Yukawa interaction is determined by the parameter $\epsilon=\left(Z /\left(1+\kappa \sigma_{c} / 2\right)\right)^{2} l_{B} / \sigma_{c}$. We neglected the higher order, effective many-body terms which are insignificant in the high salt concentration regime that is considered in this work [18. In this approximation, the effective potential (11) is the usual DLVO potential [10, 19, 20, 21, 22, 23, 24, 25]. In figure 1 an example of a typical Yukawa repulsion is given by the dot-dashed curves for $\beta \epsilon=20$ and $\kappa \sigma_{c}=100$. 
Here $\beta=1 / k T$.

Different procedures [26, 27, 28, 29, 30] exist that give the same functional form of the effective potential (II), but with a density dependent $\tilde{\kappa} \sigma_{c}=\sqrt{\left(4 \pi l_{B} \sigma_{c}^{2}\left(Z \rho_{c}+2 \rho_{s}\right)\right)}$, where $\rho_{c}$ and $\rho_{s}$ are, respectively, the densities of colloidal particles and added salt ion pairs, in the system. The density $\rho_{s}$ depends on the colloidal density and is smaller then the reservoir density due to salt exclusion or Donnan equilibrium [31, 32, 33]. For the parameters used in this work, $\beta \epsilon=20$ and $\kappa \sigma_{c}=50$, the effective $\tilde{\kappa}$ differ from the reservoir $\kappa \sigma_{c}=\sqrt{8 \pi l_{B} \sigma_{c}^{2} \rho_{s}^{r}}$ by less then $1 \%$ for a colloid density near close-packing $\rho_{s}=1.4 \sigma_{c}^{-3}$ when one applies Donnan equilibrium. Hence salt partitioning is hardly perceptible for the salt conditions considered here.

A dilute solution of polymers in a theta-solvent can be represented by (noninteracting) ideal polymers. In a theta-solvent, the attraction between the polymer segments compensates their excluded volume: the chains do not feel one another. The non-interacting polymer coils are excluded from the colloids to a centre-of-mass distance $\left(\sigma_{c}+\sigma_{p}\right) / 2$ 34]. The chains can be interpreted as freely overlapping spheres [35, 36]. The effective diameter of the polymer coil $\sigma_{p}$ is twice the depletion thickness around the colloidal particle. The pair-wise potentials in this simple model read [37]

$$
\begin{aligned}
& U_{c p}\left(\mathbf{R}_{i}-\mathbf{r}_{j}\right)= \begin{cases}\infty & \text { for }\left|\mathbf{R}_{i}-\mathbf{r}_{j}\right|<\left(\sigma_{c}+\sigma_{p}\right) / 2 \\
0 & \text { otherwise }\end{cases} \\
& U_{p p}\left(r_{i j}\right)=0
\end{aligned}
$$

Where $\mathbf{R}_{i}$ and $\mathbf{r}_{j}$ are the positions of the centres of the colloids and the polymer coils, respectively, and $r_{i j}=\left|\mathbf{r}_{i}-\mathbf{r}_{j}\right|$. We mention that the so-called Asakura-Oosawa-Vrij (AOV) model [34, 35, 36, which is a simple idealised model for colloidal hard spheres and ideal non-adsorbing polymer, is recovered by setting $\epsilon$ to zero in the effective potential (11).

However, even within the context of this highly simplified model it is often more convenient to adopt a more coarse-grained view of the binary mixture by ignoring the degrees of freedom of the polymer coils and using polymer-mediated effective interactions between the charged spheres. Below we discuss the resulting effective interactions between the charged spheres due to the pair interactions of our model.

By integrating out the degrees of freedom of the polymer coils, we can map the binary mixture of colloids and polymers onto an effective one-component system interacting with an effective one-component Hamiltonian [38, 39, 40, 41]. This effective Hamiltonian consists of zero-body, one-body, two-body, and higher-body terms. In this work, we perform Monte-Carlo simulations for the effective Hamiltonian truncated at the pairwise term, and we determine the phase behaviour of the effective one-component system. We first derive an exact expression for the polymer-mediated effective pair potential or depletion potential. In general the effective pair potential can be calculated using the generalised Gibbs adsorption equation for two colloids separated by a distance $R_{i j}$ in a sea of polymer at fixed chemical potential $\mu_{p}$, i.e., the system of colloids plus polymers is in thermodynamic equilibrium with a reservoir with only polymer chains 
(in a background solvent) at chemical potential $\mu_{p}$ [42, 43, 44, 45]

$$
\beta U_{\mathrm{dep}}\left(R_{i j}, \mu_{p}\right)=-\int_{-\infty}^{\mu_{p}} \beta d \mu_{p}^{\prime}\left(\Gamma\left(R_{i j}, \mu_{p}\right)-\Gamma\left(R_{i j}=\infty, \mu_{p}\right)\right) .
$$

The chemical potential is related to the fugacity of the polymer chains $z_{p}$ through $\beta d \mu_{p}=d \ln z_{p}$. The quantity $\Gamma\left(R_{i j}, \mu_{p}\right)$ is the adsorption or excess amount of polymer chains when the colloidal spheres are separated by a distance $R_{i j}$.

The depletion potential of the present model is identical to the depletion potential of the AOV model as the pair potentials $U_{c p}$ and $U_{p p}$ are the same in both models. Since the polymer chains do not interact, $U_{p p}=0$, we can replace $z_{p}$ by $\rho_{p}^{r}$, where $\rho_{p}^{r}$ is the polymer density in the corresponding polymer reservoir. Further within the model the depletion layers have a fixed width of $\delta \equiv \sigma_{p} / 2$ being close to the radius of gyration of a polymer chain [46, 44, 47, so the excess adsorbed amounts are directly related to the overlap volume $V_{\text {overlap }}\left(R_{i j}\right)$ of depletion layers:

$$
\Gamma\left(R_{i j}, \mu_{p}\right)-\Gamma\left(R_{i j}=\infty, \mu_{p}\right)=\rho_{p}^{r} V_{\text {overlap }}\left(R_{i j}\right)
$$

A geometrical calculation for $\sigma_{c}<R_{i j}<\sigma_{c}+\sigma_{p}$, the range where the depletion layers overlap, yields an analytic expression for $V_{\text {overlap }}\left(R_{i j}\right)$ and the depletion potential $U_{\text {dep }}$ reads for our model [34, 35, 36, 41]

$$
\begin{aligned}
& \beta U_{\text {dep }}\left(R_{i j}, \mu_{p}\right)=-\frac{\pi \sigma_{p}^{3} \rho_{p}^{r}}{6} \frac{(1+q)^{3}}{q^{3}}\left[1-\frac{3 R_{i j}}{2(1+q) \sigma_{c}}+\frac{R_{i j}^{3}}{2(1+q)^{3} \sigma_{c}^{3}}\right] \\
&=0 \quad \text { for } \sigma_{c}<R_{i j}<\sigma_{c}+\sigma_{p} \\
& \text { for } R_{i j}>\sigma_{c}+\sigma_{p}
\end{aligned}
$$

where we define the size ratio $q=\sigma_{p} / \sigma_{c}$. This Asakura-Oosawa pair potential describes an effective attraction whose depth increases linearly with the polymer density in the corresponding reservoir $\rho_{p}^{r}$. For convenience, we define the relative polymer concentration $\phi_{p}^{r} \equiv \pi \sigma_{p}^{3} \rho_{p}^{r} / 6$. Hence, $\phi_{p}^{r}=1$ defines the overlap concentration of the polymer solution. The range of the potential is given by $\sigma_{p}$. The depletion interaction $U_{\text {dep }}\left(R_{i j}\right)$ is plotted as the dashed curves for $q=0.1$ and $\phi_{p}^{r}=0.2$ in figure 1 a, for $q=0.6$ and $\phi_{p}^{r}=1$ in figure 10 and for $q=1$ and $\phi_{p}^{r}=1.4$ in figure 1r. By adjusting $q$ and $\phi_{p}^{r}$ one can manipulate the range and strength of the depletion interaction. We chose $\phi_{p}^{r}$ such that $\beta U_{\text {dep }}\left(\sigma_{c}\right)=-3.5$ for every value of $q$.

It is important to note that for sufficiently large polymer coils, effective threeand higher-body interactions can not be neglected. More precisely, we expect an increasing number of higher-body interactions to become non-zero when $q$ increases. It was shown [48 that the many-body character of the polymer-mediated effective interactions between the colloids yields a bulk phase diagram that differs substantially from those found for pair-wise simple fluids. For size ratios $q<0.1547$, three- and manybody interactions are identical to zero and the mapping of the binary mixture onto the effective one-component Hamiltonian based on pair-wise additive effective potentials is exact. 
Using the two-body approximation to the effective Hamiltonian, the total effective pair potential $U_{\text {tot }}$ reads

$$
U_{\text {tot }}\left(R_{i j}\right)=U_{c c}\left(R_{i j}\right)+U_{\text {dep }}\left(R_{i j}\right) .
$$

Examples of $U_{\text {tot }}\left(R_{i j}\right)$ are plotted in figure 1 as the full curves, which are the sums of equations (II) and (15), denoted by the dot-dashed and dashed lines, respectively. For $q=0.1$ (figure $1 \mathrm{a}$ ) there is a significant effect of the repulsive tail on the effective interaction as compared to the pure depletion contribution $U_{\text {dep }}\left(R_{i j}\right)$. There is, however, still some attraction in the $U_{\text {tot }}\left(R_{i j}\right)$ curve between the charged repulsive spheres though it is significantly reduced as compared to the pure $U_{\text {dep }}\left(R_{i j}\right)$ result. In figures $1 \mathrm{~b}$ and $1 \mathrm{c}$ the main parts of the pair interaction curves $U_{\text {tot }}\left(R_{i j}\right)$ are identical to the pure depletion part $U_{\text {dep }}\left(R_{i j}\right)$ for $q=0.6$ and $q=1$. Only the attraction at short interparticle distances is affected. The results plotted in figure 1 are qualitatively similar to the PRISM results of Ferreira et al. [16] (see their figure 5).

\section{Phase behaviour of Charged colloids}

In this section we propose a simple description for the phase behaviour of charged colloidal suspensions. We consider the screened-Coulomb repulsion as a perturbation of the hard sphere interaction which is only valid for highly screened colloidal suspensions. The effective volume fraction $\eta_{e}$ of the charged repulsive spheres is approximated as

$$
\eta_{e}=\left(\frac{\sigma_{e}}{\sigma_{c}}\right)^{3} \eta_{c}=m \eta_{c}
$$

where $\eta_{c} \equiv \pi \sigma_{c}^{3} \rho_{c} / 6$ with $\rho_{c}$ the colloid number density and $\sigma_{e}$ the effective diameter of the spheres defined by the Barker-Henderson relation [49]:

$$
\sigma_{e}=\sigma_{c}+\int_{\sigma_{c}}^{\infty}\left(1-\exp \left[-\beta U_{c c}(r)\right]\right) d r
$$

which is a useful way to generalise pair potentials of various shapes [50], in particular, perturbations from hard-sphere behaviour (see [51]). Instead of $U_{c c}$ defined in equation (11) one may use any other form for a (soft) repulsion. The physical effects are contained in the value for $m$. Since we assume that the collection of charged spheres behave similarly as a collection of pure hard spheres plus a small perturbation, we may use the Carnahan-Starling (CS) expression [52] for the Helmholtz free energy to describe the thermodynamic properties of the fluid of charged spheres:

$$
m f_{c}^{\text {fluid }}\left(\eta_{c}, T\right)=\eta_{e} \ln \eta_{e}+\frac{4 \eta_{e}^{2}-3 \eta_{e}^{3}}{\left(1-\eta_{e}\right)^{2}}-\eta_{e}+\eta_{e} \ln \frac{6 \Lambda_{c}^{3}}{\pi \sigma_{c}^{3}}
$$

where $\Lambda_{c}$ is the thermal wavelength of the colloids and where the hard sphere volume fraction $\eta_{c}$ in the classical CS expression is replaced by the effective volume fraction $\eta_{e}$, defined in equations (77) and (8). In equation (9) we use (as in [53]) the normalised Helmholtz free energy $f_{c}$, defined as $\beta F_{c} v_{c} / V$, where $F_{c}$ is the Helmholtz free energy, and $v_{c}=\pi \sigma_{c}^{3} / 6$ is the volume of a single colloid. We note that there are more advanced 
theoretical methods for Yukawa fluids [54] that describe the simulation results for longerranged repulsions.

The equation of state of the face-centred-cubic (fcc) crystal phase of pure hard spheres is described accurately by the expression proposed by Hall [55]. Likewise, the equation of state for the fcc crystal phase of the charged spheres reads

$$
m f_{c}^{\mathrm{crystal}}\left(\eta_{c}, T\right)=\eta_{e}\left(2.1306+3 \ln \left[\frac{\eta_{e}}{1-\eta_{e} / \eta_{c p}}\right]\right)+\eta_{e} \ln \frac{6 \Lambda_{c}^{3}}{\pi \sigma_{c}^{3}}
$$

containing the volume fraction at close packing $\eta_{c p}=\pi \sqrt{2} / 6 \approx 0.74$. The quantity 2.1306 is derived from computer simulation results [56].

Let us first check the accuracy of equations (9) and (10) on the level of the resulting osmotic pressure $\Pi_{c}$ that follows from $\beta \Pi_{c} v_{c}=\eta_{c} \partial f_{c} / \partial \eta_{c}-f_{c}$. The osmotic pressure for the fluid phase reads:

$$
m \beta \Pi_{c}^{\text {fluid }} v_{c}\left(\eta_{c}, T\right)=\frac{\eta_{e}+\eta_{e}^{2}+\eta_{e}^{3}-\eta_{e}^{4}}{\left(1-\eta_{e}\right)^{3}}
$$

and for the fcc crystal, the pressure is:

$$
m \beta \Pi_{c}^{\mathrm{crystal}} v_{c}\left(\eta_{c}, T\right)=\frac{3 \eta_{e}}{1-\eta_{e} / \eta_{c p}}
$$

Results using equations (11) and (12) with $U_{c c}\left(R_{i j}\right)$ given by equation (11) are plotted in figure 2 (curves) and are compared with computer simulation data (symbols), for $\beta \epsilon=20$ and $1 / \kappa \sigma_{c}$ equals 0 (full curves, open circles), 0.01 (dotted curve, filled squares) and 0.02 (dashed curve, open triangles), corresponding to $m=1,1.110$ and 1.225, respectively. The results for pressures below $\beta \Pi_{c} v_{c}=6.2$ correspond to the colloidal fluid phase, while the results for larger pressures correspond to the fcc crystal. Figure 2 shows that the pressure increases upon increasing the range of the soft repulsion. The simulation results are well described by equation (11) (see the results for $\beta \Pi_{c} v_{c}<6.2$.) for the fluid phase for $m=1, m=1.110$ and $m=1.225$. In addition, the results for the solid phase using equation (12) $\left(\beta \Pi_{c} v_{c}>6.2\right)$ agree well with the simulations for $m=1$, $m=1.110$ and $m=1.225$. Our results demonstrate that for sufficiently short-ranged soft repulsions, the screened-Coulomb interaction can be treated as a perturbation of the hard sphere potential using the Barker-Henderson relation (8).

The fluid-solid transition, first established for pure hard spheres by Alder and Wainwright [57] and Wood and Jacobson [58, can now be studied as a function of the softness of the repulsive tail. We determine the densities of the coexisting phases by equating the osmotic pressures and the chemical potentials $\beta \mu=\partial f_{c} / \partial \eta_{c}$ using equations (9) and (10). It becomes evident that the Ansatz of equations (7), (8), (9) and (10) is expected to be useful only for short-ranged soft repulsions, so for $\sigma_{e} \simeq \sigma_{c}$ or $1 / \kappa \sigma_{c} \rightarrow 0$. In order to test whether this approach is valid for relatively short-ranged soft repulsions, we compare the predicted fluid-solid transitions with computer simulation data for small values of $1 / \kappa \sigma_{c}$. In figure 3] the fluid-solid binodals calculated for $1 / \kappa \sigma_{c}<0.04$ are plotted as the full curves. In figure 3 a, the $\beta \epsilon=20$ case is considered. The dots are computer simulation results by Hynninen and Dijkstra [59]. Open symbols 
are the pure hard sphere computer simulation results of Hoover and Ree [60]. For $1 / \kappa \sigma_{c}<0.02$ the agreement is excellent though for larger Debye lengths deviations are found. This deviation is not surprising since the simple theoretical method is based on a perturbation from the hard sphere system and is, hence, expected to be only accurate for very small values of $1 / \kappa \sigma_{c}$. Still, this simple theory for charged colloidal sphere dispersions suffices our purpose of studying the stability of charged colloid-polymer mixtures in the regime of $1 / \kappa \sigma_{c}<0.02$. To be more specific, this means that our Ansatz describes well the case of colloidal particles with a diameter of, say, $\sigma_{c}=100$ $\mathrm{nm}$ with a Debye length smaller than about $2 \mathrm{~nm}$ or, equivalently, an ionic strength $>0.02 M$ (in the case of monovalent ions in water at room temperature). Actually, in nature, the ionic strength in aqueous salt concentrations is usually above $0.02 M$, so for large spheres we still capture the biologically relevant ionic strength regime. For globular proteins we can only compare with experimental data at significant salt concentrations but we can still make estimates of the main trends.

In figure [3], we compare our theoretical results (full curves) with computer simulation data (crosses) for $\beta \epsilon=39$. Again, our Ansatz is in fair agreement with the computer simulation results (data points) [59]. Hence we validated our approach to describe the fluid and fcc crystal equations of state and the fluid-solid transition of a charged sphere dispersion for $1 / \kappa \sigma_{c}<0.02$. This provides a base for studying the effect of adding non-adsorbing polymer to such a suspension.

\section{Phase behaviour of Charged colloid-polymer mixtures}

\subsection{Free volume theory}

We study the stability of a mixture of charged colloidal spheres and non-adsorbing polymer chains in a common (background) solvent. A simple approach that successfully describes the stability of polymer-colloid mixtures is the semi-grand canonical free volume or osmotic equilibrium theory [3, 61]. In this approach, a macroscopic volume $V$ at temperature $T$ is considered, that contains $N_{c}$ colloids together with polymer chains and solvent, which are in osmotic equilibrium with a reservoir containing only solvent and polymer chains. Hence, the system is considered in the $\left(N_{c}, V, z_{p}, T\right)$ ensemble, in which the number of colloids $N_{c}$ and the fugacity of the polymer chains $z_{p}$ are fixed. Consider the thermodynamic identity [41]:

$$
\beta F\left(N_{c}, V, z_{p}\right)=\beta F\left(N_{c}, V, z_{p}=0\right)+\int_{0}^{z_{p}} d z_{p}^{\prime}\left(\frac{\partial \beta F\left(N_{c}, V, z_{p}{ }^{\prime}\right)}{\partial z_{p}{ }^{\prime}}\right)_{z_{p}{ }^{\prime}}
$$

where we dropped the temperature dependence for convenience. The integrand can now be Taylor expanded about $z_{p}=0$ :

$$
\begin{aligned}
\beta F\left(N_{c}, V, z_{p}\right)= & \beta F\left(N_{c}, V, z_{p}=0\right)+z_{p}\left(\frac{\partial \beta F\left(N_{c}, V, z_{p}\right)}{\partial z_{p}}\right)_{z_{p}=0} \\
& +\mathcal{O}\left(z_{p}^{2}\right)+\cdots
\end{aligned}
$$


where the partial derivative can be written as

$$
\left(\frac{\partial \beta F\left(N_{c}, V, z_{p}\right)}{\partial z_{p}}\right)_{z_{p}=0}=\left(\frac{\partial F}{\partial \mu_{p}}\right)_{z_{p}=0} \frac{\partial \beta \mu_{p}}{\partial z_{p}}=-\frac{\left\langle N_{p}\right\rangle_{z_{p}=0}}{z_{p}} .
$$

The number of polymer chains $\left\langle N_{p}\right\rangle_{z_{p}=0}$ can be related by definition to the averaged free volume that is available for the polymer chains $\left\langle V_{\text {free }}\right\rangle_{z_{p}=0}$ in the system of spheres that is undistorted by the addition of polymers:

$$
\left\langle N_{p}\right\rangle_{z_{p}=0} \equiv \rho_{p}^{r}\left\langle V_{\text {free }}\right\rangle_{z_{p}=0} .
$$

where $\rho_{p}^{r}$ is the density of ideal polymer in the corresponding reservoir. Defining the free volume fraction $\alpha \equiv\left\langle V_{\text {free }}\right\rangle_{z_{p}=0} / V$, we can rewrite equation (13) as:

$$
\beta F\left(N_{c}, V, z_{p}\right)=\beta F\left(N_{c}, V, z_{p}=0\right)-\alpha \rho_{p}^{r} V+\mathcal{O}\left(z_{p}^{2}\right)+\cdots
$$

The free volume theory [3] retains only the first-order term, neglecting terms $\mathcal{O}\left(z_{p}^{2}\right)$ and higher. With this assumption the normalised thermodynamic potential $f \equiv \beta F v_{c} / V$ can be written as the sum of two terms:

$$
f\left(N_{c}, V, z_{p}\right)=f_{c}\left(N_{c}, V\right)-\alpha \phi_{p}^{r} q^{-3}
$$

The first term in equation (18) is the normalised Helmholtz free energy $f\left(N_{c}, V, z_{p}=\right.$ $0) \equiv f_{c}\left(N_{c}, V\right)$ of a 'pure colloid' fluid at a given $\eta_{c}$, while the second can be interpreted as a perturbation due to the presence of polymer chains. Note that equation (13)-(18) holds for any colloid-polymer and polymer-polymer interactions within the assumptions that are made. However, for interacting polymers, a different approximation of the free volume theory gives a better description of the experimental phase diagrams [53]. All information about the interactions between colloid and polymer is contained in the variation of $\alpha$ with $\eta_{c}$. For our model and the AOV model, the free volume fraction $\alpha$ can be calculated accurately from scaled particle theory [62, 3] (see e.g. Meijer 63] for a comparison with computer simulation results). Once the coexisting colloid volume fractions are determined for given $\phi_{p}^{r}$, the actual relative polymer concentrations can be obtained in the coexisting phases from $\phi_{p} \equiv \alpha \phi_{p}^{r}$. An expression for the free volume fraction available for the polymer chains in a mixture of charged spheres using scaled particle theory reads

$$
\alpha=\left(1-\eta_{c}\right) \exp \left(-A \gamma-B \gamma^{2}-C \zeta-3 C \zeta^{2}-3 C \zeta^{3}\right),
$$

where $\gamma=\eta_{c} /\left(1-\eta_{c}\right), \zeta=\eta_{e} /\left(1-\eta_{e}\right), A=3 q+3 q^{2}, B=9 q^{2} / 2$, and $C=q^{3}$. An explicit derivation is given in Appendix 1. Equation (19) reduces to the classical expression of Lekkerkerker et al. [3] for the case $\eta_{e}=\eta_{c}(m=1)$. The free volume available for the polymer chains is thus mainly a function of the pure hard sphere volume fraction $\eta_{c}$; it is only affected by the screened-Coulomb repulsion at high colloid volume fractions or large polymer chains. In the derivation of equation (19) an equal statistical weight is assigned to all (non-overlapping) hard-sphere configurations, whereas the weight should involve the polymer-mediated effective interactions and the screenedCoulomb interactions. We expect our results to be accurate only if $\sigma_{e} / \sigma_{c}-1<<q$. If the depletion layers become small compared to $\sigma_{e}-\sigma_{c}$ one expects hardly any overlap 
of depletion layers. In figure 4 we compare the result of the free volume fraction $\alpha$ as a function of the colloid volume fraction $\eta_{c}$ of equation (19) with Monte Carlo simulation results for $\left\langle\rho_{p}\right\rangle_{z_{p}} / \rho_{p}^{r}$ using equation (42) for $q=0.1$. It is worth mentioning that $\alpha$ is evaluated in the pure charged colloid system in the free volume theory, i.e., $z_{p}=0$, while $\left\langle\rho_{p}\right\rangle_{z_{p}} / \rho_{p}^{r}$ from simulations do depend on $z_{p}$. In the simulations, however, we use $z_{p}$ along the bulk binodals as shown in figure 11 (which will be discussed later). The technical details of the simulations are described in Appendix 2. The theoretical curves in figure 4 show only a slight effect of the screened-Coulomb repulsion on $\alpha$ for $\eta_{c}>0.4$. Within the statistical error bars, no significant effect of the screened-Coulomb repulsion was detected in the simulations on the free volume fraction. Moreover, the $z_{p}$-dependence is hardly noticeable. We thus conclude that equation (19) is accurate for short-ranged screened-Coulomb repulsions for $q=0.1$ or, equivalently, that the effect of the screened-Coulomb repulsion on $\alpha$ is negligible.

We can now analyse the effect of the screened-Coulomb repulsion on the phase behaviour. The osmotic pressures $\Pi$ and colloid chemical potentials $\mu_{c}$ can be found by differentiation of the Helmholtz free energy (18). The colloid volume fractions in each of the coexisting phases, $\eta_{c}^{1}$ and $\eta_{c}^{2}$, are obtained by equating $\mu_{c}$ and $\Pi$ at fixed polymer reservoir concentration $\phi_{p}^{r}$. The Helmholtz free energy density (10) is used for the solid phase, while equation (9) is employed for the fluid. The free volume theory predicts for the AOV model, i.e., a system of colloidal hard spheres and ideal polymer, a broadening of the fluid-solid transition with increasing $\phi_{p}^{r}$ for size ratios $q<0.3$, while the fluid-fluid transition is metastable with respect to a broad fluid-solid transition [3, 41].

We now turn to our case of charged colloidal spheres and ideal polymer chains. In figure 5, we plot the phase diagram using the free volume theory (18) for a size ratio $q=0.1$ and $\left(\kappa \sigma_{c}\right)^{-1}=0.0,0.005,0.01$, and 0.0125 in the $\left(\eta_{c}, \phi_{p}^{r}\right)$ plane. For $\left(\kappa \sigma_{c}\right)^{-1}=0$ and $\phi_{p}^{r}=0$, we recover the well-known pure hard sphere freezing transition at $\eta_{c}=0.494$ and 0.545 60. In the case of charged spheres, the freezing transition at $\phi_{p}^{r}=0$ shifts to lower colloid volume fractions $\eta_{c}$, which is in line with the results in figure 3 , and is due to a larger effective volume of the charged spheres. Figure 5 shows clearly that the fluidsolid transition widens upon increasing the polymer concentration. More specifically, the broadening of the freezing transition shifts to higher $\phi_{p}^{r}$ with increasing range of the screened-Coulomb repulsion $\left(\kappa \sigma_{c}\right)^{-1}$. This can be explained as follows. Upon increasing the range of the screened-Coulomb repulsion, $\eta_{e}=m \eta_{c}$, and hence $f_{c}$, increases. At the same time the free volume fraction $\alpha$ is not affected significantly (see figure 4) upon adding a screened-Coulomb repulsion. So, in order to attain a similar effect on $f$ (see Eq. (18)), a higher polymer concentration is required to broaden the freezing transition.

For larger values of $q$, say $q>0.4$, a fluid-fluid coexistence becomes stable in the AOV model, which dominates the phase behaviour at colloid volume fractions $\eta_{c}<0.49$. In analogy with figures 1b and 1 we choose $q=0.6$ and 1 and study the effect of the repulsive screened-Coulomb interaction on the fluid-solid and fluid-fluid transition. In figures [6] and [7, we plot the predictions from free volume theory in the $\left(\eta_{c}, \phi_{p}^{r}\right)$ representation for $q=0.6$ and $q=1.0$, respectively. We again find that the 
freezing transition at $\phi_{p}^{r}=0$ shifts to lower colloid volume fractions $\eta_{c}$ upon increasing $\left(\kappa \sigma_{c}\right)^{-1}$. In addition, figures 6 and 7 show that the fluid-fluid demixing shifts to higher $\phi_{p}^{r}$ with increasing range of the screened-Coulomb repulsion $\left(\kappa \sigma_{c}\right)^{-1}$. Hence, the trends are similar as for $q=0.1$. The screened-Coulomb repulsion reduces the depletion effect. The critical points are indicated as the filled circles in figure [6] and 7 and they indicate that the critical colloid volume fraction $\eta_{c}$ shifts to somewhat smaller values upon increasing the range of the soft repulsion.

Finally, we convert the polymer reservoir concentration $\phi_{p}^{r}$ to that in the actual system $\phi_{p}$. Figure 8 shows the conversion of the phase diagram of figure 5 for $q=0.1$ and $\beta \epsilon=20$ and Debye screening lengths $\left(\kappa \sigma_{c}\right)^{-1}=0$ (dashed curves; the pure hard sphere case), $\left(\kappa \sigma_{c}\right)^{-1}=0.005$ (dot-dashed curves), $\left(\kappa \sigma_{c}\right)^{-1}=0.01$ (full curves) and 0.0125 (dotted curves). The phase stability of a mixture of charged colloids and neutral polymer chains in an aqueous salt solution is thus expected to depend very sensitively on the screening length, and thus on the salt concentration, at least for small size ratios q. In figure 9, we investigate the effect of $\beta \epsilon$ on the phase behaviour. We plot the converted phase diagram for the same set of parameters as in figure 8, i.e., $q=0.1$ and varying Debye screening lengths $\left(\kappa \sigma_{c}\right)^{-1}$, but with $\beta \epsilon=39$ instead of $\beta \epsilon=20$. There is a striking similarity between the two sets of results and, hence, we conclude that the effect of $\beta \epsilon$ is not significant. For larger values of $\beta \epsilon$, the system becomes more sensitive to $\kappa \sigma_{c}$ and thus to the salt concentration. Finally, figure 10 shows the conversion of the phase diagram as shown in figure[7 for $q=1.0$ and $\beta \epsilon=20$ and Debye screening lengths $\left(\kappa \sigma_{c}\right)^{-1}=0$ (dashed curves; the pure hard sphere case), $\left(\kappa \sigma_{c}\right)^{-1}=0.01$ (full curves) and 0.02 (dotted curves).

It follows that an increase of the reduced Debye length $\left(\kappa \sigma_{c}\right)^{-1}$ shifts the fluid-fluid coexistence curves upwards. Using a PRISM approach, Ferreira et al. [16] also found this trend for the spinodal curve of the demixing fluid (see their figure 11) based on determining the composition where the inverse structure factor vanishes in the long wavelength limit. Figures [7 and [10] show clearly that the shift in polymer concentration of the fluid-fluid binodals for $q=1$ is weak compared to the shift in the fluid-solid binodals for small $q$.

A relevant quantity that measures the relative influence of the screened-Coulomb repulsive pair interaction is $\left(\kappa \sigma_{p}\right)^{-1}$ or $\left(\kappa \sigma_{c} q\right)^{-1}$. Hence, the size of the polymer chains (or the depletion thickness) as compared to the range of the repulsion determines the relative importance of the screened-Coulomb repulsion on the total effective depletion interaction. In biological systems such as charged proteins mixed with neutral polysaccharides, where often $\kappa^{-1}<\sigma_{c} q$, we expect that the phase behaviour is only moderately sensitive to the salt concentration. Decreasing the salt concentration significantly is then expected to stabilise the charged biocolloid dispersion against depletion-induced demixing. This explains the enhanced miscibility found in mixtures of proteins mixed with neutral non-adsorbing polysaccharides in aqueous salt solutions [6. In many charged colloidal dispersions the soft repulsion is expected to suppress the depletion effect. In several applications such as paints and food dispersions where 
colloidal particles are mixed with polymer chains, a screened-Coulomb repulsion helps stabilising the dispersion.

\subsection{Simulations using the effective Hamiltonian}

In order to test the validity of the predictions from free volume theory, we compare our results with Monte Carlo simulations. We determine the phase diagram of the effective one-component system by calculating the dimensionless free energy density $f=\beta F v_{c} / V$ as a function of the colloid packing fraction $\eta_{c}$ and the fugacity of the polymer chains $z_{p}$ in simulations. For non-interacting chains the fugacity $z_{p}$ equals the density of polymer chains $\rho_{p}^{r}$ in the corresponding reservoir. As the free energy density cannot be measured directly in a Monte Carlo simulation, we use thermodynamic integration to relate the free energy of the effective system to that of a reference system at the same colloid volume fraction $\eta_{c}$. To this end, we write the total free energy density as the sum of two contributions

$$
f\left(N_{c}, V, z_{p}\right)=f_{c}\left(N_{c}, V\right)+f_{\mathrm{dep}}\left(N_{c}, V, z_{p}\right),
$$

where $f_{c}\left(N_{c}, V\right)$ is the free energy density for a system of $N_{c}$ charged colloids in a volume $V$ interacting with a hard-core repulsive Yukawa potential (11), and $f_{\text {dep }}$ is the contribution of the depletion potential (5) to the free energy density. The free energy density $f_{c}\left(N_{c}, V\right)$ is computed using the so-called $\lambda$-integration for the fluid phase and the standard Frenkel-Ladd method for the solid phase 64 with the Einstein crystal as a reference. A second $\lambda$-integration is then carried out, for both the fluid and the solid phase, to determine the free energy density contribution $f_{\text {dep}}$. For more details of the simulations, we refer the reader to Appendix 2. In order to map out the phase diagram we determine the total free energy density $f\left(\eta_{c}, z_{p}\right)$ for many state points $\left(\eta_{c}, z_{p}\right)$ in simulations. We employ common tangent constructions at fixed $z_{p}$ to obtain the coexisting phases [40]. In order to assess the performance of the free volume theory, phase diagrams were determined for some of the parameters as in figure $5(q=0.1)$. The results are shown in figure 11 and agree semi-quantitatively with those in figure 5. The main difference is due to the fact that the results of both approaches deviate already for the AOV model (hard sphere colloids with ideal polymer, i.e., $\left(\kappa \sigma_{c}\right)^{-1}=0$ ) 41]. For the AOV model, the theoretical binodal is shifted with a factor of about 1.3 in $\phi_{p}^{r}$ compared to the simulations. This factor between the theoretical predictions and the simulation results is about 1.2 when the screened-Coulomb repulsion is added.

We also compare the free volume theory results for the phase behaviour for $q=0.6$ and 1 as shown in figures [ 6 and $[$ with computer simulations. The phase diagrams obtained from Monte Carlo simulations of the effective one-component systems are

plotted in figure [5] $(q=0.6)$ and figure 5 ( $(q=1.0)$. The main theoretical trends are also found in our simulation results. Again, the data of the simulations suggest that a higher polymer concentration is required to induce the fluid-fluid transition upon increasing the range of the soft repulsion. 
Finally, we stress that the free volume theory incorporates some of the many-body effects which are present at large $q$, while our simulations are based on a two-body approximation to the effective Hamiltonian. It is therefore difficult to make a direct comparison between the simulation results and those obtained from free volume theory. However, for $q \leq 0.1547$, the mapping of the charged colloid-polymer mixture onto an effective one-component Hamiltonian based on depletion pair potentials is exact and thus a direct comparison is feasible for our results for $q=0.1$.

\section{Concluding remarks}

We have studied the effect of a short-ranged screened-Coulomb repulsion on the phase stability of mixtures containing charged spheres and non-adsorbing polymer chains. The charged spheres are described as hard spheres with an additional screened-Coulomb or Yukawa repulsion with the screening length given by the Debye length $\kappa^{-1}$, setting the range of the soft repulsion. The phase behaviour of the charged sphere dispersion is described using standard expressions for the colloidal hard sphere fluid and fcc crystal with the hard sphere volume fraction replaced by an effective volume fraction that depends on the Yukawa interaction between the spheres. Our results obtained from free volume theory and Monte Carlo simulations show that the additional screened-Coulomb repulsion reduces the depletion effect. For mixtures of small polymers plus relatively large charged spheres the fluid-solid transition is shifted to significantly larger polymer concentrations with increasing Debye screening length $\kappa^{-1}$, while for relatively larger polymers the effect is weaker: the resulting fluid-fluid binodal is affected weakly by adding a short-ranged soft repulsion. In general, the range of the screened-Coulomb repulsion compared to the range of the depletion attraction determines qualitatively the reduction of the depletion effect, and hence, shifts the fluid-fluid and fluid-solid binodals correspondingly. In conclusion, a mixture of charge-stabilised colloids and non-adsorbing polymers at large concentrations of both components can be stabilised by lowering the salt concentration, which increases the range of the screened-Coulomb interaction of the colloids.

\section{Acknowledgments}

This work was supported by the SoftComp Network of Excellence. We thank M. Schmidt, A.-P. Hynninen, H.N.W. Lekkerkerker, A. Vrij, G. A. Vliegenthart and J. Buitenhuis for useful discussions. We thank the Dutch National Computer Facilities foundation for access to the SGI Origin 3800 and SGI Altix 3700. 


\section{Appendix 1: Free volume fraction in a charged sphere dispersion}

In this appendix we consider the free volume fraction $\alpha$ that is available for ideal polymer chains in a sea of charged spheres with diameter $\sigma_{c}$. As the centre-of-mass of the polymer chains is excluded from the centre-of-mass of the charged colloids by a distance $\left(\sigma_{c}+\sigma_{p}\right) / 2$ and the polymer interactions are ideal, $\alpha$ is just the free volume fraction for a single hard sphere with diameter $\sigma_{p}$ in a sea of charged spheres. This free volume fraction $\alpha$ can be determined from the chemical potential of the polymer chains. The chemical potential for inserting a polymer in a sea of charged spheres consists of an ideal gas term and a work term $W$.

$$
\beta \mu_{p}=\ln \rho_{p} \Lambda_{p}^{3}+W
$$

Following Widom's particle insertion method [65], the required work to insert a polymer $W$ is equal to $\beta W=-\ln \alpha$. The work $W$ can be determined from scaled particle theory, that considers two limits. In this theory, the size of the particle is scaled with a parameter $x$. In the limit $x \ll 1$, the polymer coils reduce to points, and hence the volume fraction available to the polymer is simply unity minus the sum of the overall hard sphere volumes plus the depletion layers around them:

$$
\alpha(x \ll 1)=1-\frac{\pi}{6} \rho_{c}\left(\sigma_{c}+x \sigma_{p}\right)^{3}
$$

where $\rho_{c}=N_{c} / V$ is the number of colloidal spheres per volume (related to $\eta_{c}$ via $\left.\eta_{c}=\pi \rho_{c} \sigma_{c}^{3} / 6\right)$. Hence, it follows

$$
\beta W(x \ll 1)=-\ln \left[1-\frac{\pi}{6} \rho_{c}\left(\sigma_{c}+x \sigma_{p}\right)^{3}\right]
$$

On the other hand, if $x \gg 1$, the work required to insert a polymer coil in a sea of charged spheres, is approximately the work to create a hole with the size of the polymer coil, which is equal to the volume of the polymer coil times the osmotic pressure $\Pi_{c}$ of the dispersion of charged colloids:

$$
W(x \gg 1)=\frac{\pi}{6} x^{3} \sigma_{p}^{3} \Pi_{c} .
$$

In scaled particle theory, $W(x \ll 1)$ is expanded about $x=0$ up to order $x^{2}$ and $W(x \gg 1)$ is added as the $x^{3}$ term.

$$
W(x)=W(x=0)+x\left(\frac{\partial W}{\partial x}\right)_{x=0}+\frac{1}{2} x^{2}\left(\frac{\partial^{2} W}{\partial x^{2}}\right)_{x=0}+\frac{\pi}{6} x^{3} \sigma_{p}^{3} \Pi_{c}(25)
$$

Scaling the polymer coils to the desired size by $x=1$, yields

$$
\begin{aligned}
\beta W(x=1)= & -\ln \left[1-\eta_{c}\right]+3 q \gamma+\frac{1}{2}\left(6 q^{2} \gamma+9 q^{2} \gamma^{2}\right) \\
& +\frac{\pi}{6} \sigma_{p}^{3} \beta \Pi_{c}
\end{aligned}
$$

where $\gamma=\eta_{c} /\left(1-\eta_{c}\right)$. Hence $\alpha$ follows straightforwardly from $W \equiv W(x=1)$. For pure hard spheres one usually takes the Percus-Yevick result for the pressure $\Pi_{H S}$ from the virial route (see [51]) since it is consistent with SPT

$$
\frac{\beta \Pi_{H S}}{\rho_{c}}=\frac{1+\eta_{c}+\eta_{c}^{2}}{\left(1-\eta_{c}\right)^{3}}=\frac{1}{1-\eta_{c}}+\frac{3 \eta_{c}}{\left(1-\eta_{c}\right)^{2}}+\frac{3 \eta_{c}^{2}}{\left(1-\eta_{c}\right)^{3}}
$$


Inserting this expression for $\Pi_{H S}$ into equation 26 for $\Pi_{c}$ yields

$$
\beta w=-\ln \left[1-\eta_{c}\right]+(A+C) \gamma+(B+3 C) \gamma^{2}+3 C \gamma^{3}
$$

where $A, B$, and $C$, are defined below equation [19, Hence, we arrive at the standard SPT result for the free volume fraction of ideal polymer in a sea of hard spheres [3]

$$
\alpha=\left(1-\eta_{c}\right) \exp \left[-\left((A+C) \gamma+(B+3 C) \gamma^{2}+3 C \gamma^{3}\right)\right]
$$

In case of colloidal spheres interacting with a Yukawa pair potential, we rewrite equation (27) following the approach outlined in section 3 giving a pressure

$$
\frac{\beta \Pi_{Y u k}}{\rho_{c}}=\frac{1+\eta_{e}+\eta_{e}^{2}}{\left(1-\eta_{e}\right)^{3}}=\frac{1}{1-\eta_{e}}+\frac{3 \eta_{e}}{\left(1-\eta_{e}\right)^{2}}+\frac{3 \eta_{e}^{2}}{\left(1-\eta_{e}\right)^{3}}
$$

where $\eta_{e}$ is defined by equation (77). Using this expression for $\Pi_{c}$ in equation 26 yields equation 19

\section{Appendix 2: Technical details of the simulations}

This section describes the technical details of the simulations. To this end we consider

the total effective one-component Hamiltonian of the colloids at fixed polymer fugacity $z_{p}$

$$
H\left(z_{p}\right)=H_{c c}+H_{\text {dep }}\left(z_{p}\right)
$$

where the Hamiltonian $H_{c c}$ consists of a sum of colloid-colloid pair potentials $U_{c c}$, which can be split into a sum of hard-sphere potentials $U_{H S}$ and a sum of repulsive Yukawa potentials:

$$
H_{c c}=\sum_{i<j}^{N_{c}} U_{c c}\left(R_{i j}\right)=\sum_{i<j}^{N_{c}} U_{H S}\left(R_{i j}\right)+\sum_{i<j}^{N_{c}} U_{Y u k}\left(R_{i j}\right)
$$

with

$$
U_{Y u k}\left(R_{i j}\right)=\epsilon \frac{\exp \left(\kappa \sigma_{c}\left(R_{i j} / \sigma_{c}-1\right)\right)}{R_{i j} / \sigma_{c}} \quad \text { for } \quad R_{i j}>\sigma_{c}
$$

and $H_{\text {dep }}$ a sum of depletion potentials $U_{\text {dep }}$ (5):

$$
H_{\text {dep }}\left(z_{p}\right)=\sum_{i<j}^{N_{c}} U_{\text {dep }}\left(R_{i j} ; z_{p}\right) \text {. }
$$

The different Hamiltonians give rise to the corresponding free energy contributions of equation (20).

To determine the free energy contribution of the Yukawa potential, for the fluid phase, we introduce the auxiliary Hamiltonian

$$
H_{c c, \lambda}^{\text {fluid }}=\sum_{i<j}^{N_{c}} U_{H S}\left(R_{i j}\right)+\lambda \sum_{i<j}^{N_{c}} U_{Y u k}\left(R_{i j}\right)
$$

where $0 \leq \lambda \leq 1$ is a dimensionless coupling parameter: at $\lambda=0$ the auxiliary Hamiltonian is that of a pure system of $N_{c}$ hard spheres, while at $\lambda=1$ it is the 
Hamiltonian of $N_{c}$ charged spheres. The free energy is determined by applying the standard $\lambda$-integration technique 64

$$
f_{c}\left(N_{c}, V\right)=f_{H S}\left(N_{c}, V, \lambda=0\right)+\frac{v_{c}}{V} \int_{0}^{1} d \lambda\left\langle\sum_{i<j}^{N_{c}} \beta U_{Y u k}\left(R_{i j}\right)\right\rangle_{N_{c}, V, \lambda} .
$$

The angular brackets denote a canonical average over a system of $N_{c}$ particles interacting with the Hamiltonian $H_{c c, \lambda}^{\text {fluid }}$, while $f_{H S}\left(N_{c}, V, \lambda=0\right)$ is the free energy of a system of hard spheres, for which we use the Carnahan-Starling expression [52]. We start the canonical simulations from a random, non-overlapping configuration, and use 15000 MC sweeps per particle for equilibration and typically 15000 production moves for each value of the coupling parameter $\lambda$. In principle, the free energy of the solid phase can be computed with the same technique using the Hall expression for the free energy of the hard sphere crystal [55]. However the latter is only properly defined for packing fractions larger than the value at hard sphere freezing $\eta_{c}=0.545$, while charged spheres can yield crystal phases at lower packing fractions. A different technique is used for the solid phase by introducing the auxiliary Hamiltonian

$$
H_{c c, \lambda}^{\text {solid }}=\sum_{i<j}^{N_{c}} U_{c c}\left(R_{i j}\right)+\lambda k_{B} T \sum_{i=1}^{N_{c}}\left(\mathbf{r}_{i}-\mathbf{r}_{o, i}\right)^{2} / \sigma_{c}^{2}
$$

where $\mathbf{r}_{o, i}$ denote the ideal lattice position of particle $i$ in a fcc crystal. The free energy is computed by applying the integration technique introduced by Frenkel and co-workers [56, 66

$$
\begin{aligned}
f_{c}\left(N_{c}, V\right)= & f_{\text {ein }}^{C M}\left(N_{c}, V, \lambda=\lambda_{\max }\right)+f_{\text {corr }}\left(N_{c}, V\right) \\
& -\frac{v_{c}}{V} \int_{0}^{\lambda_{\max }} d \lambda\left\langle\sum_{i=1}^{N_{c}}\left(\mathbf{r}_{i}-\mathbf{r}_{o, i}\right)^{2} / \sigma_{c}^{2}\right\rangle_{\lambda}^{C M},
\end{aligned}
$$

where the angular brackets denote a canonical average of the mean square displacement of $N_{c}$ particles interacting with the Hamiltonian $H_{c c, \lambda}^{\text {solid }}$, while the superscript $C M$ denotes that it is calculated for a crystal with fixed centre of mass. The parameter $\lambda_{\max }$ is chosen such that for $\lambda=\lambda_{\max }$ the system behaves like a non-interacting Einstein crystal with fixed centre of mass and Madelung energy $U_{Y u k}\left(\mathbf{r}_{0}^{N_{c}}\right)$, i.e., the potential energy of a crystal with all particles at their ideal lattice positions. Typical values for $\lambda_{\max }$ range from 1000 to 100000 for high densities. The free energy of a non-interacting Einstein crystal with fixed centre of mass reads

$$
\begin{aligned}
f_{\text {ein }}^{C M}\left(N_{c}, V, \lambda=\lambda_{\max }\right)= & \frac{v_{c}}{V} \beta U_{Y u k}\left(\mathbf{r}_{0}^{N_{c}}\right)-\frac{3\left(N_{c}-1\right) v_{c}}{2 V} \ln \left[\frac{\pi}{\lambda_{\max }}\right] \\
& +\frac{\left(N_{c}-1\right) v_{c}}{V} \ln \left[\frac{\Lambda_{c}^{3}}{\sigma_{c}^{3}}\right]
\end{aligned}
$$

The correction term $f_{\text {corr }}$ arises when the constraint on the centre of masses is released, i.e., the Helmholtz free energy difference between the unconstrained and constrained crystal:

$$
f_{\text {corr }}\left(N_{c}, V\right)=\frac{v_{c}}{V} \ln \left[\frac{\Lambda^{3}}{V N_{c}^{1 / 2}}\right]
$$


The equilibration is done for $15000 \mathrm{MC}$ steps per particle, and the averages are taken for $15000 \mathrm{MC}$ steps per particle.

To determine the free energy contribution of the AOV depletion potential $f_{\text {dep }}$ we employ a second thermodynamic integration for the solid and fluid phase:

$$
\begin{aligned}
f_{\text {dep }} & =f\left(N_{c}, V, \rho_{p}^{r}\right)-f\left(N_{c}, V, \rho_{p}^{r}=0\right) \\
& =\int_{0}^{\rho_{p}^{r}} d \rho_{p}^{r \prime}\left(\frac{\partial f\left(N_{c}, V, \rho_{p}^{r \prime}\right)}{\partial \rho_{p}^{r \prime}}\right)_{N_{c}, V, \rho_{p}^{r \prime}}
\end{aligned}
$$

The system at $\rho_{p}^{r}=0$ is a system of colloids interacting with pair potentials $U_{c c}$. The integral is calculated by dividing the interval $\left[0, \rho_{p}^{r}\right]$ in 20 to 30 equally spaced intervals. We used $10000 \mathrm{MC}$ steps per particles for equilibration, while averages were taken for 20000 MC steps per particle. In addition we used the integrand to determine the number density of ideal polymer in the system using the thermodynamic relation

$$
\left(\frac{\partial f\left(N_{c}, V, \rho_{p}^{r}\right)}{\partial \rho_{p}^{r}}\right)=\left(\frac{\partial f\left(N_{c}, V, z_{p}\right)}{\partial z_{p}}\right)=-v_{c} \frac{\left\langle\rho_{p}\right\rangle_{\rho_{p}^{r}}}{\rho_{p}^{r}}
$$

\section{References}

[1] P.R. Sperry, H.B. Hopfenberg, and N.L. Thomas. J. Colloid Interface Sci., 82:62, 1981.

[2] A.P. Gast, C.K. Hall, and W.B. Russel. J. Colloid Interface Sci., 96:251, 1983.

[3] H. N. W. Lekkerkerker, W. C. K. Poon, P. N. Pusey, A. Stroobants, and P. B. Warren. Europhys. Lett., 20:559, 1992.

[4] S. M. Ilett, A. Orrock, W. C. K. Poon, and P. N. Pusey. Phys. Rev. E, 51:1344, 1995.

[5] W.C.K. Poon. J. Phys: Condens. Matter, 14:R859, 2002.

[6] V. Ya. Grinberg and V. B. Tolstoguzov. Food Hydrocolloids, 11:145, 1997.

[7] J.-L. Doublier, C. Garnier, C. Renard, and C. Sanchez. Curr. Opin. Colloid Interface Sci., 5:184, 2000.

[8] A.K. Van Helden, J.W. Jansen, and A. Vrij. J. Colloid Interface Sci., 81:354, 1981.

[9] P. N. Pusey and W. Van Megen. Nature, 320:340, 1986.

[10] E.J.W. Verwey and J. Th. Overbeek. Theory of the Stability of Lyophobic Colloids. Elsevier, Amsterdam, 1948.

[11] T.T. Hebert. Phytopathology, 53:362, 1963.

[12] S. Finet and A. Tardieu. J. Crystal Growth, 232:40, 2001.

[13] M. Casselyn, J. Perez, A. Tardieu, P. Vachette, J. Witz, and H. Delacroix. Acta Cryst. D, 57:1799, 2001.

[14] P.D. Patel and W.B. Russel. J. Colloid Interface Sci., 131:192, 1989.

[15] O. Annunziata, N. Asherie, A. Lomakin, J. Pande, O. Ogun, and G.B. Benedek. PNAS, 99:14165, 2002.

[16] P.G. Ferreira, M. Dymitrowska, and L. Belloni. J. Chem. Phys., 113:9849, 2000.

[17] A.R. Denton and M. Schmidt. J. Chem. Phys., 122:244911, 2005.

[18] R. van Roij, M. Dijkstra, and J.P. Hansen. Physical Review E, 59:2010, 1999.

[19] P. Pieranski. Contemp. Phys., 24:25, 1983.

[20] M.O. Robbins, K. Kremer, and G.S. Grest. J. Chem. Phys., 88:3286, 1993.

[21] W.B. Russel, D.A. Saville, and W.R. Schowalter. Colloidal Dispersions. Cambridge Universtity Press, USA, 1999.

[22] H. Löwen and G. Kramposthuber. Europhys. Lett., 23:673, 1993.

[23] M.J. Stevens and M.O. Robbins. J. Chem. Phys., 98:2319, 1993.

[24] M. Dijkstra. Curr. Opin. Colloid Interface Sci., 4:372, 2001. 
[25] C. Russ, H.H. von Grunberg, M. Dijkstra, and R. van Roij. Phys. Rev. E, 66:011402, 2002.

[26] H. Graf and H. Löwen. Physical Review E, 57:5744, 1998.

[27] A.R. Denton. Physical Review E, 62:3855, 2000.

[28] P.B. Warren. J. Chem. Phys., 112:4683, 2000.

[29] L. Belloni. J. Phys.: Condens. Matter, 12:R549, 2000.

[30] M. Dijkstra and R. van Roij. J. Phys: Condens. Matter, 10:1219, 1998.

[31] F.G. Donnan and A.B. Harris. J. Chem. Soc., 99:1554, 1911.

[32] F.G. Donnan. Z. Electrochem., 17:572, 1911.

[33] F.G. Donnan. Chem. Rev., 1:73, 1924.

[34] S. Asakura and F. Oosawa. J. Pol. Sci., 33:183, 1958.

[35] A. Vrij. Pure Appl. Chem., 48:471, 1976.

[36] H. De Hek and A. Vrij. J. Colloid Interface Sci., 84:409, 1981.

[37] We note that in the original paper of Asakura and Oosawa 34 the polymers were regarded as pure hard spheres in the dilute limit. Vrij [35, 36] modelled the polymer chains as freely overlapping spheres with $U_{p p}=0$.

[38] M. Dijkstra, R. van Roij, and R. Evans. Phys. Rev. Lett., 81:2268, 1998.

[39] M. Dijkstra, R. van Roij, and R. Evans. Phys. Rev. Lett., 82:117, 1999.

[40] M. Dijkstra, R. van Roij, and R. Evans. Phys. Rev. E, 59:5744, 1999.

[41] M. Dijkstra, J. M. Brader, and R. Evans. J. Phys: Condens. Matter, 11:10079, 1999.

[42] D.G. Hall. J. Chem. Soc., Faraday Trans., 68:2169, 1972.

[43] S.G. Ash, D.H. Everett, and C. Radke. J. Chem. Soc., Faraday Trans., 69:1256, 1973.

[44] R. Tuinier, G.A. Vliegenthart, and H.N.W. Lekkerkerker. J. Chem. Phys., 113:10768, 2000.

[45] R. Tuinier, D.G.A.L. Aarts, H.H. Wensink, and H.N.W. Lekkerkerker. Phys. Chem. Chem. Phys., $5: 3707,2003$.

[46] E. Eisenriegler. J. Chem. Phys., 79:1052, 1983.

[47] G.J. Fleer, A.M. Skvortsov, and R. Tuinier. Macromolecules, 36:7857, 2003.

[48] M. Dijkstra and R. van Roij. Phys. Rev. Lett., 89:208303, 2002.

[49] J.A. Barker and D. Henderson. J. Chem. Phys., 47:4714, 1967.

[50] A.A. Louis and R. Roth. J. Phys: Condens. Matter, 13:L777, 2001.

[51] J.-P. Hansen and I.R. McDonald. Theory of Simple Liquids. Academic Press, San Diego, CA, USA, 1986.

[52] N.F. Carnahan and K.E. Starling. J. Phys. Chem., 51:635, 1969.

[53] D.G.A.L. Aarts, R. Tuinier, and H.N.W. Lekkerkerker. J. Phys: Condens. Matter, 14:7551, 2002.

[54] T.W. Cochran and Y.C. Chiew. J. Chem. Phys., 121:1480, 2004.

[55] C.K. Hall. J. Chem. Phys., 52:2252, 1972.

[56] D. Frenkel and A.J.C. Ladd. J. Chem. Phys., 81:3188, 1984.

[57] B.J. Alder and T.E. Wainwright. J. Chem. Phys., 27:1208, 1957.

[58] W.W. Wood and J.D. Jacobson. J. Chem. Phys., 27:1207, 1957.

[59] A-P. Hynninen and M. Dijkstra. Phys. Rev. E, 68:021407, 2003.

[60] W.G. Hoover and F.M. Ree. J. Chem. Phys., 49:3609, 1968.

[61] H. N. W. Lekkerkerker. Colloids and Surfaces, 51:419, 1990.

[62] J.L. Lebowitz, E. Helfand, and E. Praestgaard. J. Chem. Phys., 43:774, 1965.

[63] E. J. Meijer and D. Frenkel. J. Chem. Phys., 100:6873, 1994.

[64] D. Frenkel and B. Smit. Understanding Molecular Simulation 2nd edition, volume 1 of Computational Science Series. Academic Press, 2002.

[65] B. Widom. Science, 157:375, 1967.

[66] J. M. Polson, E. Trizac, S. Pronk, and D. Frenkel. J. Chem. Phys., 112:5339, 2000. 

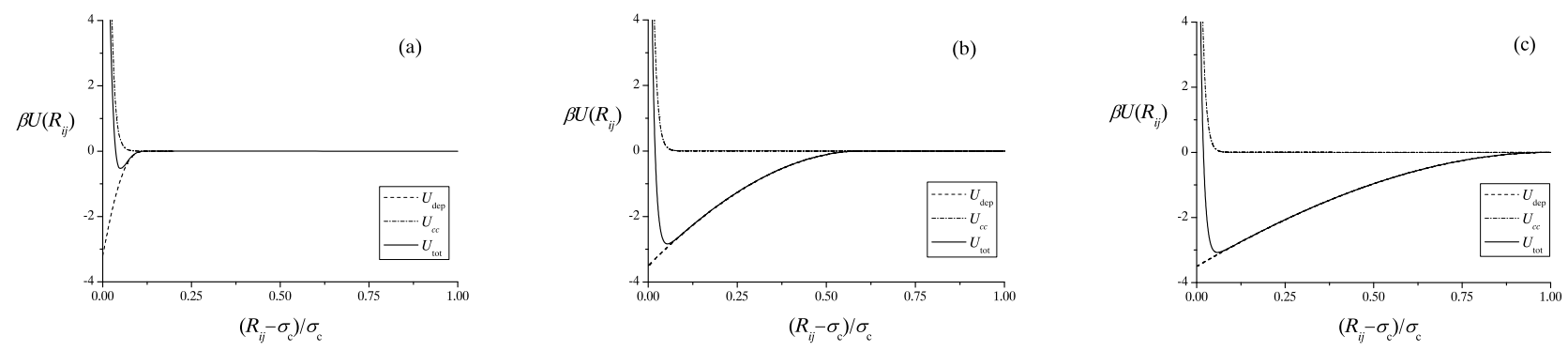

Figure 1. Pair interaction between two colloidal spheres with hard-core diameter $\sigma_{c}$ interacting with a Yukawa pair potential $U_{c c}$ (equation 1) and an effective depletion interaction $U_{\text {dep }}$ (equation 5) due to the presence of non-adsorbing polymer coils with an effective diameter $\sigma_{p}$. The total effective interaction $U_{\text {tot }}$ (equation 6) is denoted by the full curve. The Yukawa repulsion is characterised by $\beta \epsilon=20$ and $\kappa \sigma_{c}=100$. The depletion interaction are: a) for a size ratio $q=\sigma_{p} / \sigma_{c}=0.1$ and relative polymer concentration $\phi_{p}^{r}=0.2$, b) $q=0.6$ and $\phi_{p}^{r}=1$, and c) $q=1$ and $\phi_{p}^{r}=1.4$.

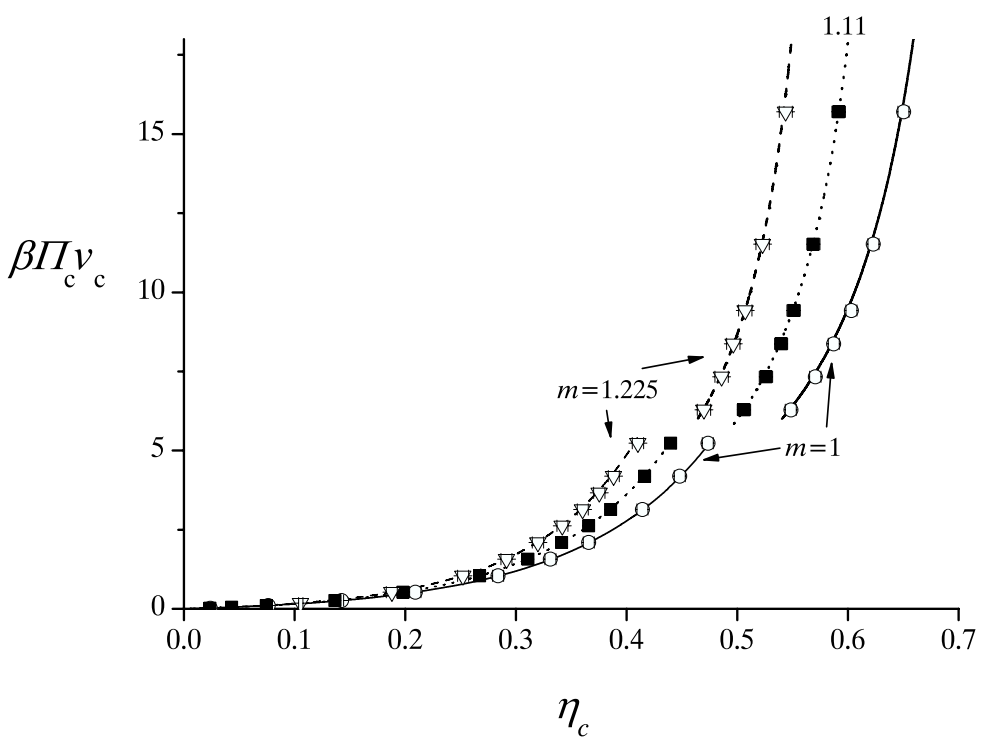

Figure 2. Pressure of a dispersion of charged colloidal spheres interacting with $U_{c c}\left(R_{i j}\right)$ (see equation (10) for $\beta \epsilon=20$ and $1 / \kappa \sigma_{c}=0$ (open circles, full curves), 0.01 (filled squares, dotted curves), and 0.02 (open triangles, dashed curves), corresponding to $m=1,1.110$, and 1.225, respectively, in equation (17). The symbols denote the simulation results, while the curves denote the theoretical predictions of equations (11) (lower set of pressures) and (12) (upper set). 

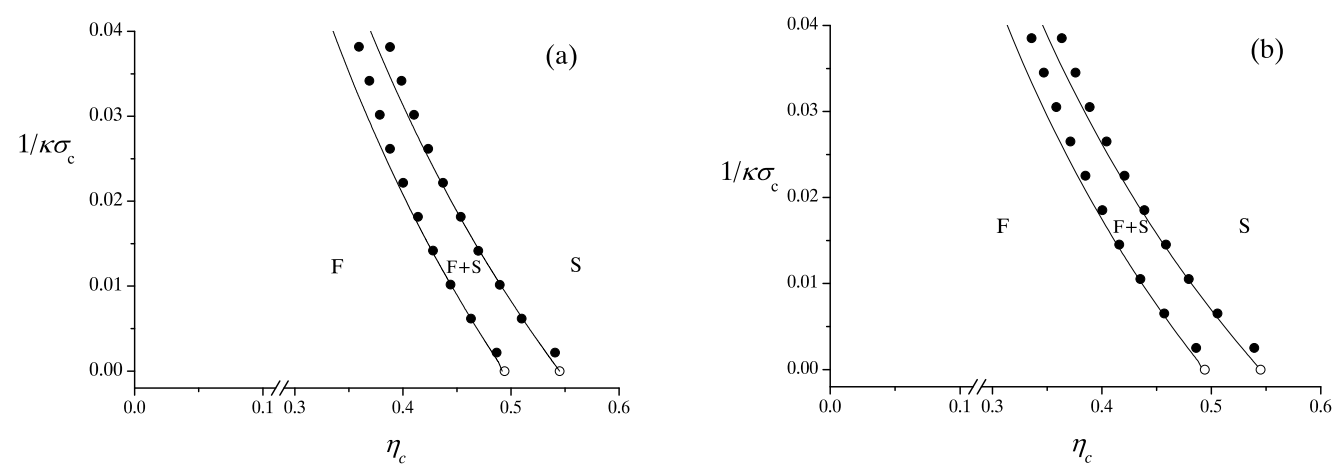

Figure 3. Fluid-solid (fcc) transition of charged colloidal spheres interacting with a hard-core repulsive Yukawa potential (1) with a) $\beta \epsilon=20$ and b) $\beta \epsilon=39$. Open symbols denote simulation results of pure hard spheres taken from Ref. 60]. Filled circles denote the simulation results of Hynninen and Dijkstra [59]. Full curves correspond to the theoretical predictions as described in section 3

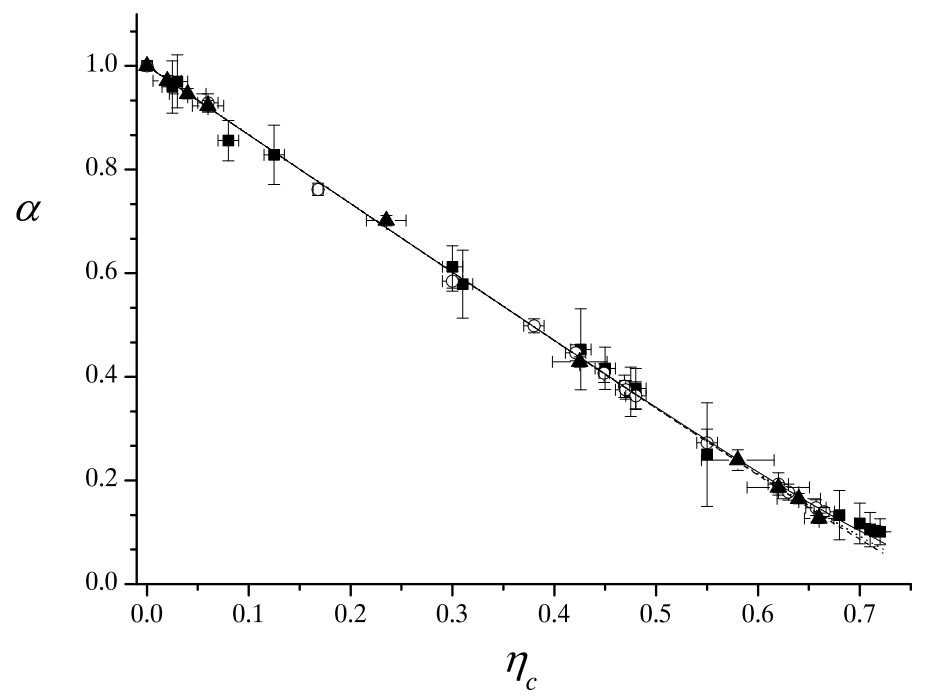

Figure 4. Free volume fraction $\alpha \equiv\left\langle V_{\text {free }}\right\rangle_{z_{p}=0} / V$ for a mixture of charged colloids and ideal polymer with size ratio $q=\sigma_{p} / \sigma_{c}=0.1$ as a function of hard-core volume fraction $\eta_{c}$. The screened-Coulomb repulsion is characterised by $\beta \epsilon=20$ and various values of $\kappa \sigma_{c}$. Full, dotted and dashed curves represent equation (19) for $\kappa \sigma_{c}=\infty(m=1), 100(m=1.110)$ and $80(m=1.138)$, respectively. Note that the differences in the theoretical curves are only noticeable at high $\eta_{c}$. The symbols with errorbars denote the Monte Carlo simulation results for $\left\langle\rho_{p}\right\rangle_{z_{p}} / \rho_{p}^{r}$ using equation (42) for $\kappa \sigma_{c}=\infty$ (closed squares), $\kappa \sigma_{c}=100$ (open circles) and $\kappa \sigma_{c}=80$ (closed triangles), where we used $z_{p}$ at bulk coexistence (see figure 11). 


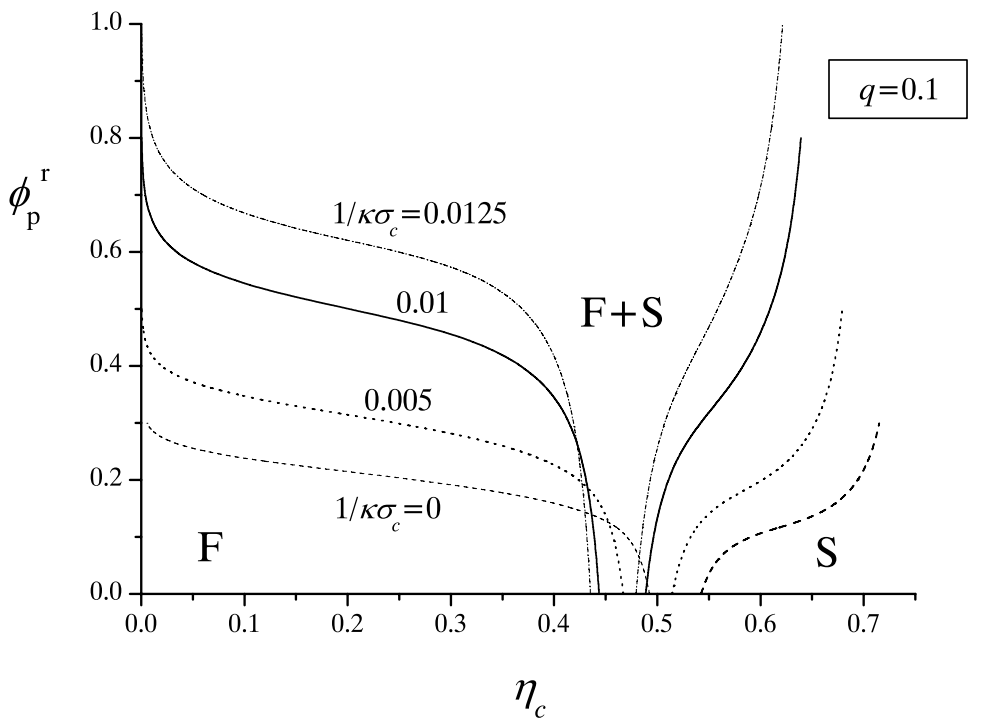

Figure 5. Phase diagram of a mixture of charged spheres and ideal polymer as obtained from free volume theory for $q=0.1$ as a function of the colloid volume fractions $\eta_{c}$ and the ideal polymer reservoir concentration $\phi_{p}^{r}$. The screened-Coulomb repulsion (11) is characterised by $\beta \epsilon=20$ and various values of $\kappa \sigma_{c}$ as indicated. $F$ and $S$ denote the stable fluid and solid fcc phase. $F+S$ denotes the stable fluid-solid coexistence region.

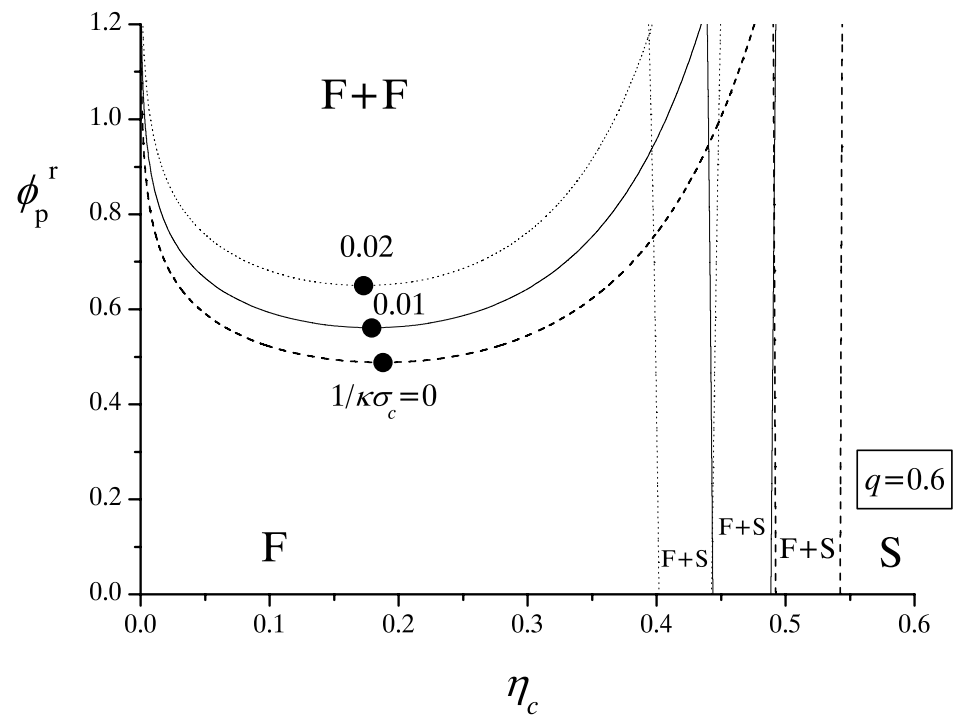

Figure 6. Same as figure 5 but for $q=0.6 . \quad F+F$ denotes the stable fluid-fluid coexistence region. 


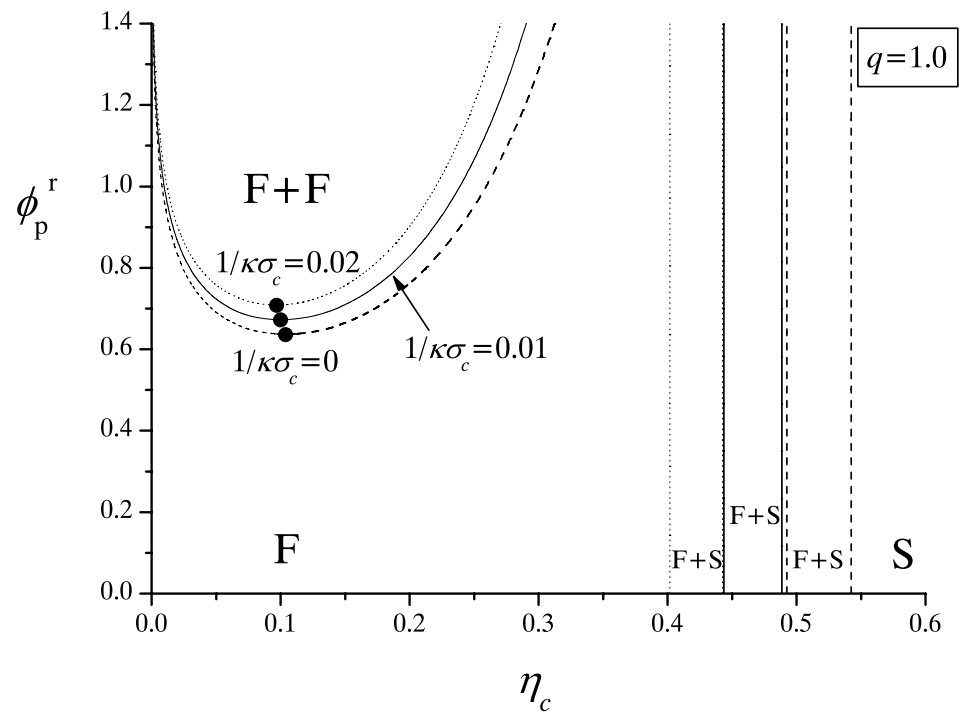

Figure 7. Same as figure 5 but for $q=1.0 . \quad F+F$ denotes the stable fluid-fluid coexistence region.

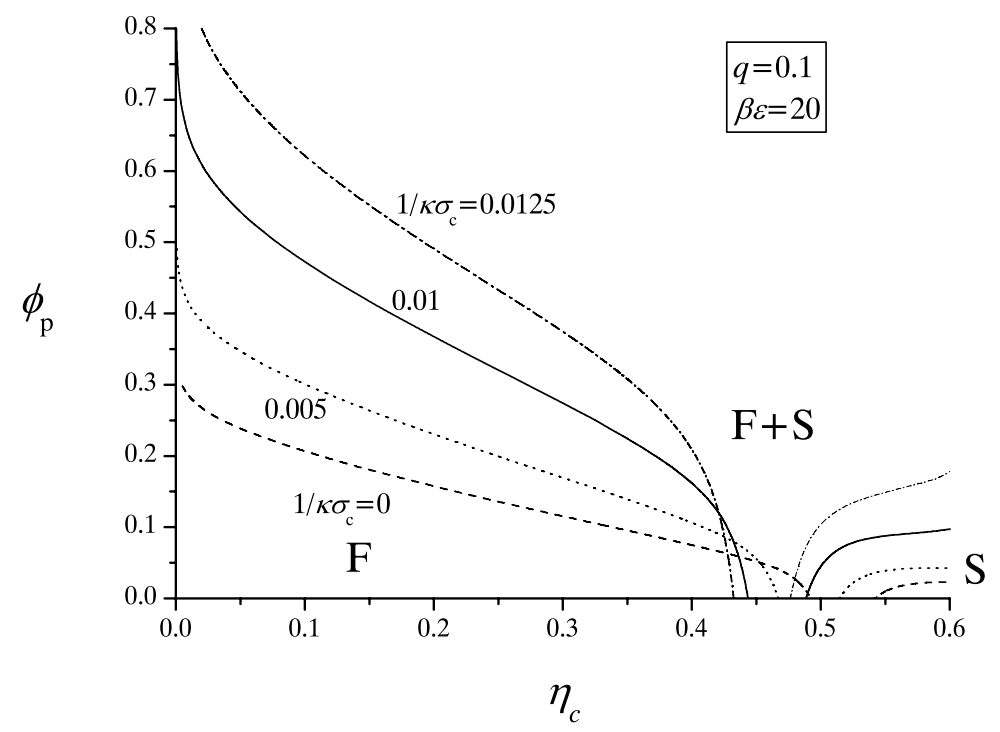

Figure 8. Same as figure 5 but as a function of the actual ideal polymer concentration $\phi_{p}$. 


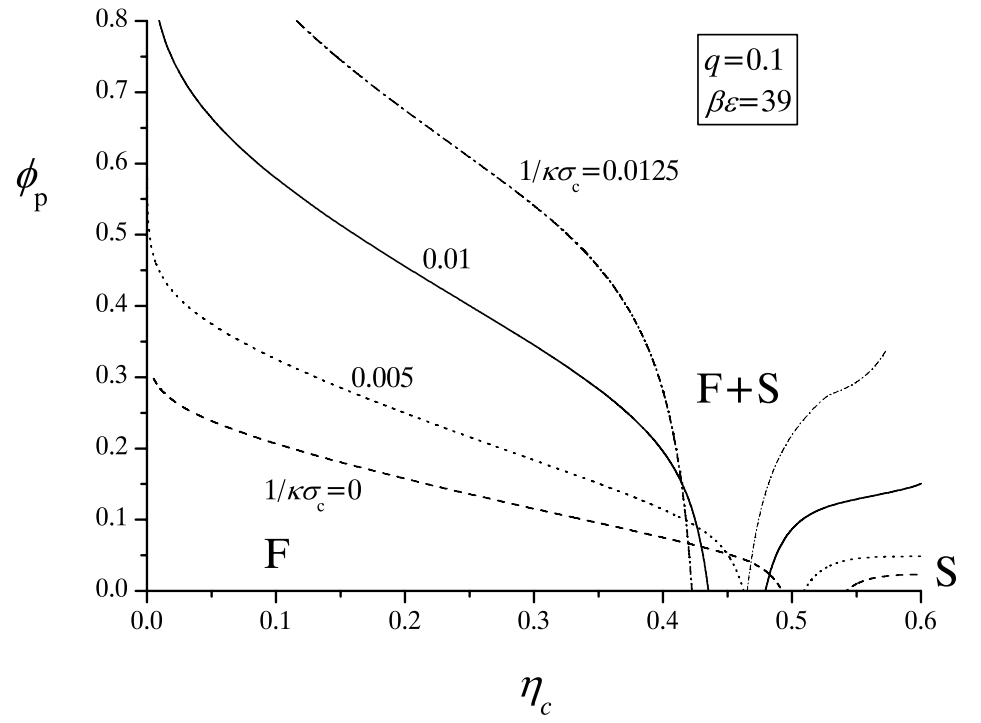

Figure 9. Same as figure 8 but with $\beta \epsilon=39$.

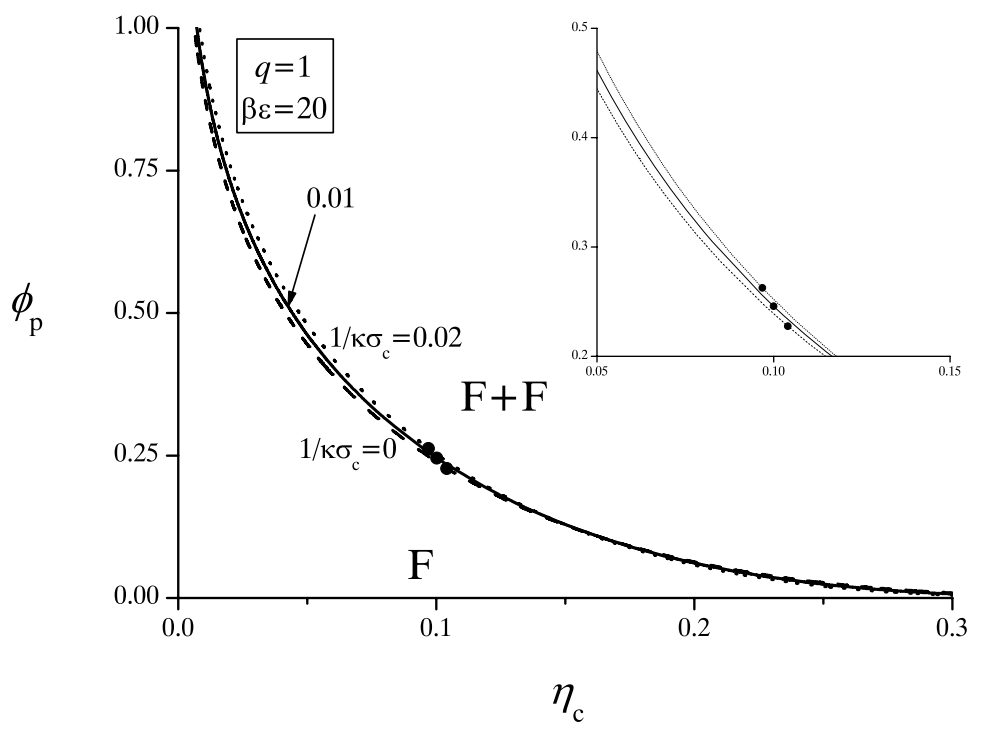

Figure 10. Same as in figure 8 but with size ratio $q=1.0$. The filled circles indicate the location of the gas-liquid critical points. The inset is a blow-up of the critical region of the liquid-gas binodal. 


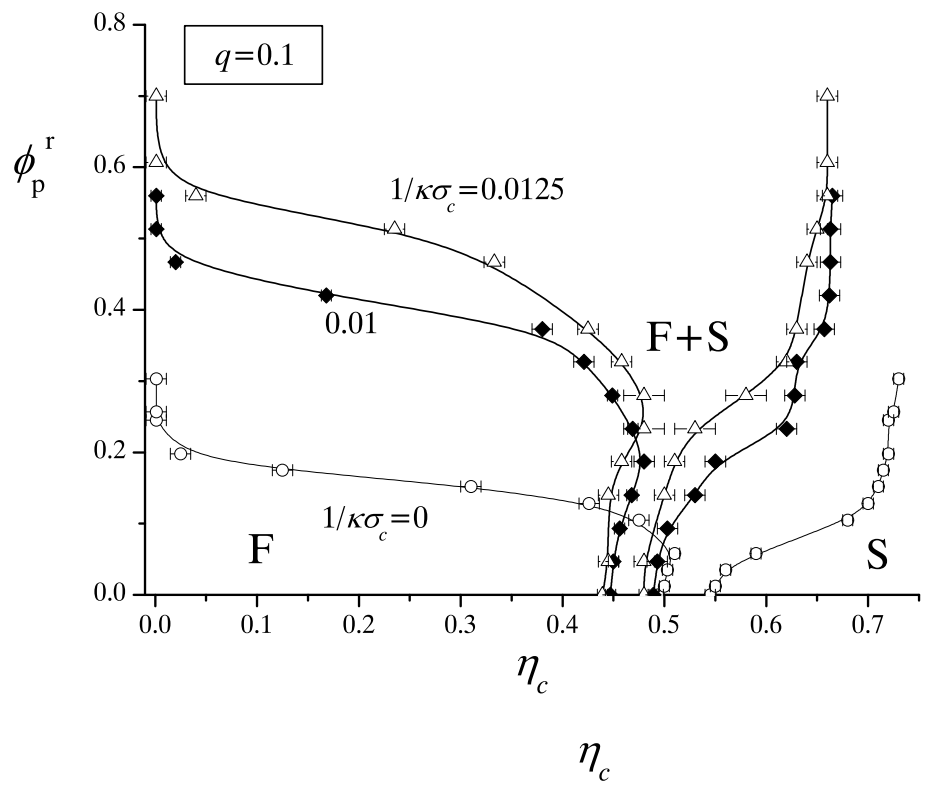

Figure 11. Phase diagram of a mixture of charged spheres and ideal polymer as obtained from simulations of the effective pair potential (6) for $q=0.1$ as a function of the colloid volume fractions $\eta_{c}$ and the ideal polymer reservoir concentration $\phi_{p}^{r}$. The screened-Coulomb repulsion (1) is characterised by $\beta \epsilon=20$ and various values of $\kappa \sigma_{c}$ as indicated. The curves serve as a guide to the eye. Open circles correspond to the pure hard sphere case $\left(\kappa \sigma_{c}\right)^{-1}=0$, closed circles to a screened-Coulomb repulsion with $\left(\kappa \sigma_{c}\right)^{-1}=0.01$ and open triangles to $\left(\kappa \sigma_{c}\right)^{-1}=0.0125$. $F$ and $S$ denote the stable fluid and solid fcc phase. $F+S$ denotes the stable fluid-solid coexistence region. 


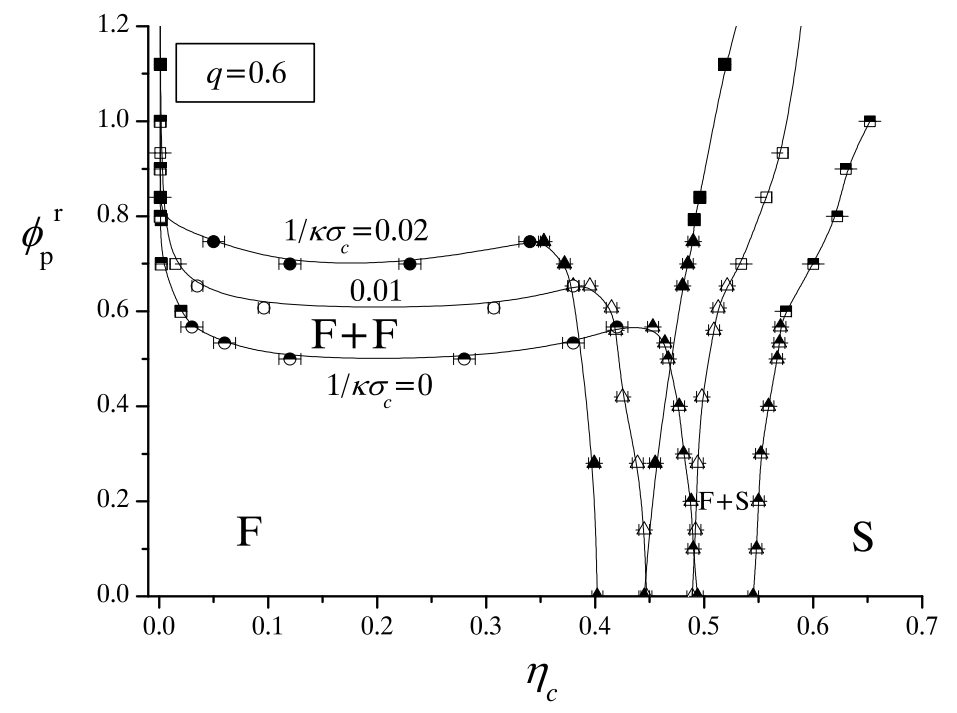

Figure 12. Same as figure 11 but for $q=0.6 . F+F$ denotes the stable fluid-fluid coexistence region.

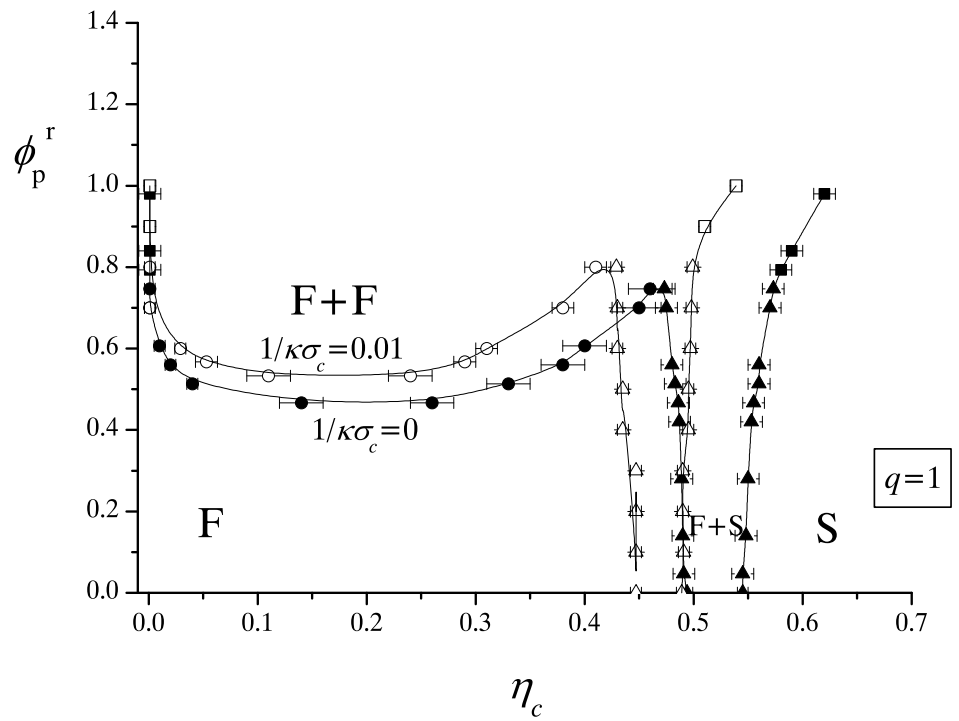

Figure 13. Same as figure [1] but for $q=1 . \quad F+F$ denotes the stable fluid-fluid coexistence region. 